\title{
Mucinous adenocarcinoma of the renal pelvis
}

\author{
K. JosHI \\ M.D.
S. MATHUR
M.D.

\author{
K. JAIN \\ M.D. \\ G. C. Mehrotra \\ M.D.
}

Department of Pathology, Medical College, Rohtak-124001 (Haryana), India

\section{Summary}

A case of mucinous adenocarcinoma of the renal pelvis occurring in association with staghorn calculus and severe pyelonephritis is reported. The incidence and aetiopathogenesis of this neoplasm is briefly discussed.

\section{Introduction}

Most malignant renal pelvic tumours are epithelial in origin and can be divided into transitional cell carcinoma, squamous cell carcinoma and adenocarcinoma. Mucinous adenocarcinoma of the renal pelvis is a rare entity. Twenty-eight cases from Western literature were tabulated by Aufderheide and Streitz (1974) and 3 have been reported in the Indian literature (Saxena, Sanghal and Bhargava, 1966; Wahal et al., 1966; Guha et al., 1975). The tumour is associated with long-standing obstruction and infection, and possibly originates from metaplastic endothelium (Liwincz et al., 1975). In view of the relative rarity of this tumour, a case is being reported, where this unusual tumour was encountered in association with nephrolithiasis.

\section{Case report}

A 50-year-old man presented with pyuria and thinning of the urinary stream for 8 months. He had developed a high grade fever with chills and rigors 8 months before, which lasted for 6 days. There had been frequency and burning during micturition for the last 2 months. General physical examination revealed nothing positive. Abdominal examination revealed a mass palpable in the left lumbar region, extending to the left hypochondrium. It was firm, smooth surfaced, tender on deep pressure, bimanually palpable and moving with respiration. Per rectum examination disclosed a moderately enlarged prostate.

Routine haematological investigations were within normal limits. Urine examination revealed $8-10$ pus cells/high power field and the culture grew Escheri- \% chia coli with a count of $7 \times 10^{6} / \mathrm{ml}$. Blood sugar and blood urea were $4.5 \mathrm{mmol} / \mathrm{l}$ and $4.98 \mathrm{mmol} / \mathrm{l}_{+}$ respectively. Intravenous pyelography showed a staghorn calculus in the left renal area, with a non-음 functioning left kidney.

At operation the left kidney was found to be veryc large and lobulated. On aspiration of the renal pelvis, a thick, whitish pus was obtained. A left $\vec{\bullet}$ renal nephrectomy was performed.

\section{Pathology}

Gross examination

The kidney measured $20 \times 11 \times 9 \mathrm{~cm}$ and was of a⿳亠二口犬

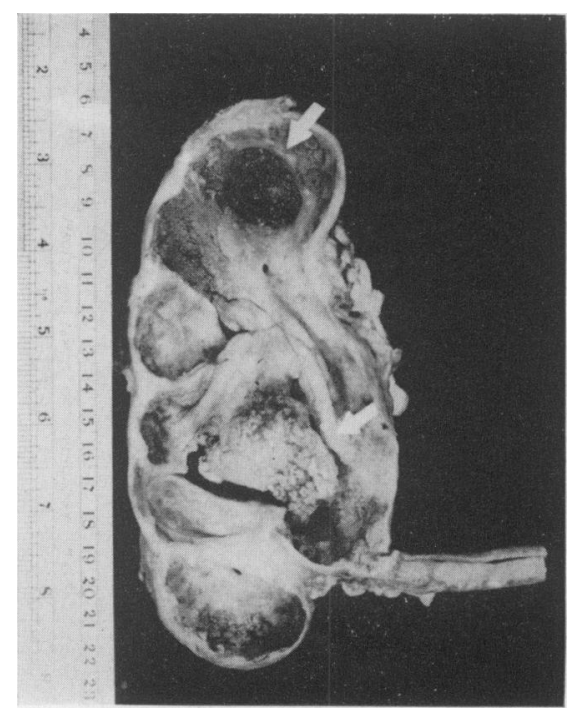

FIG. 1. Cut surface of the kidney showing part of the staghorn calculus in the minor calyx (upper arrow) and papillary tumour in the renal pelvis (lower arrow). 
cystic appearance. The external surface was lobulated. The cut surface revealed a markedly dilated pelvi-calyceal system with thinning of the renal parenchyma. The calyces were filled with sanguinous pus and mucoid material. A staghorn calculus was seen at the pelvis. The pelvis and the major calyces showed a finely papillary and mucoid tumour lining the mucosa. The minor calyces had a rough and coarsely granular surface.

\section{Microscopic examination}

The pelvic and calyceal mucosa was altered and transformed into papillary structures, with gland formation, lined by vacuolated goblet cells, similar to that of the large bowel. These cells forming the glandular structures showed hyperchromatism, atypism and multi-layering. At many areas, there was formation of signet-ring cells with abundant pools of mucin. There was also invasion of tumour glands into the renal parenchyma, and an intense inflammatory reaction. The interstitium was infiltrated by lymphocytes and plasma cells, and formation of lymphoid follicles. The glomeruli showed sclerosis and periglomerular fibrosis. Tubules were atrophic.

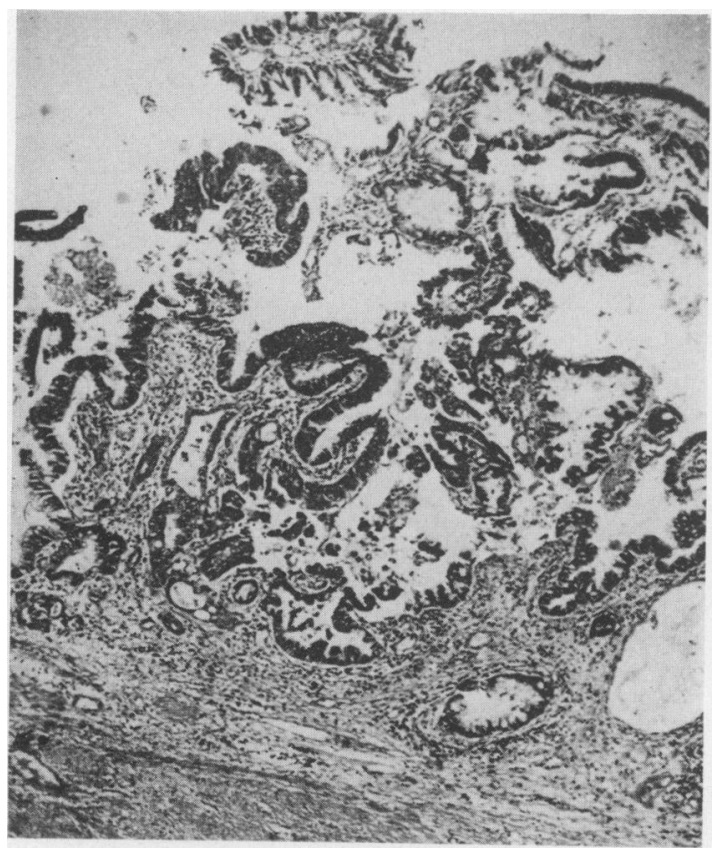

FIG. 2. Mucin-secreting adenocarcinoma of the renal pelvis showing the papillary nature of the tumour and infiltration of the wall $(\mathrm{HE}, \times 122 \cdot 5)$.

\section{Discussion}

The incidence of renal pelvic tumours has been variably recorded in the literature. It varies from $7 \cdot 7 \%$ (Lucke and Schlumberger, 1957) to $3 \cdot 1 \%$ (Sirsat, 1968) of renal cancers. Most of the renal pelvic tumours are epithelial in origin and can be divided histologically into transitional cell carcinoma, squamous cell carcinoma and adenocarcinoma. Amongst these, mucinous adenocarcinoma of the renal pelvis is distinctly rare (Ragin and Rolnick, 1950).

Twenty-eight cases of mucinous adenocarcinoma of the renal pelvis have been reviewed and tabulated by Aufderheide and Streitz (1974). The authors emphasize the importance of strictly adhering to the criteria of malignancy, so that instances of mucinous metaplasia may not be confused with adenocarcinoma. These criteria are: (1) Histological evidence of architectural or cellular atypism, (2) Microscopic evidence of invasion of renal pelvic wall, renal parenchyma, nodes or distant metastases. (3) Evidence of overt invasion, recurrence, nodal or distant metastases.

The first 2 criteria were fulfilled in the present case.

Only 3 cases of mucinous adenocarcinoma have so far been described in the Indian literature (Saxena et al., 1966; Wahal et al., 1966; Guha et al., 1975).

Metaplasia in urinary tract epithelium is usually squamous, but the occurrence of metaplastic mucinsecreting cells is well recognized. It is commonly found in the mucosa in exstrophy of the bladder, but rarely in other conditions (Gordon, 1963). True intestinal differentiation, namely brush border striations, Paneth's and argentaffin cells have also been noted in the metaplastic renal pelvic epithelium of a case of recurrent calculi (Gordon, 1963). Prominent among the aetiological factors contributing to metaplastic changes in the urothelium are long-standing bacterial infections, obstructive uropathies and the presence of calculi. The other factors include avitaminosis $A$, toxic metabolic products and specific carcinogens (MacLean and Fowler, 1956).

Most cases of mucinous adenocarcinoma of the renal pelvis are associated with chronic longstanding infection and calculi (Aufderheide and Streitz, 1974); thus, it has been postulated that these tumours, like those of the urinary bladder, originate from metaplastic urothelium (Liwniz et al., 1975).

Most of these cases are clinically undetected and are diagnosed from the surgically resected specimen. Since the tumour occupies a large area of the pelvis and calyces, and is exfoliated into the urinary tract, urinary cytology may be used as a diagnostic aid. 


\section{References}

Aufderheide, A.C. \& Streitz, J.M. (1974) Mucinous adenocarcinoma of the renal pelvis-report of two cases. Cancer, New York, Philadelphia, etc., 33, 167.

GoRDon, A. (1963) Intestinal metaplasia of urinary tract epithelium. Journal of Pathology and Bacteriology, 85, 441.

Guha, P., Datta, B.N., Aikat, B.K. \& Bannerji, A.K. (1975) Tumours of renal pelvis. Indian Journal of Pathology and Bacteriology, 18, 21.

Liwnicz, B.H., LePow, H., Schutte, H., Fernandez, R. \& Caberwal, D. (1975) Mucinous adenocarcinoma of the renal pelvis - discussion of possible pathogenesis. Journal of Urology, 114, 306.

LuCKe, B. \& SCHLumberger, H.S. (1957) Tumor of the kidney, renal pelvis and ureter. In: Atlas of Tumor Pathol- ogy. Fascicle VIII - 30. Armed Forces Institute of Pathology, Washington, D.C.

Maclean, J.T. \& Fowler, V.B. (1956) Pathology of tumors of the renal pelvis and ureter. Journal of Urology, 75, 384. @

RaGiN, A.B. \& RolniCK, H.C. (1950) Mucus-producing $\subseteq$ adenocarcinoma of the renal pelvis. Journal of Urology, $\vec{\Rightarrow}$ 63, 66.

Saxena, O., Sanghal, B.C. \& Bhargava, K.N. (1966) Primary adenocarcinoma of renal pelvis: review of literature with first case report from India. Indian Journal of $\overline{\bar{G}}$ Pathology and Bacteriology, 9, 344.

SiRSAT, M.V. (1968) Pathology of primary malignant neo- $\mathbb{\mathbb { Q }}$ plasmas of the kidney. Indian Journal of Cancer, 5, 37.

Wahal, K.M., Rastogi, K.M., Mehrotra, R.M.L. \& Rai, œ S.S. (1966) Mucus-producing adenocarcinoma of renal $\overrightarrow{0}$ pelvis. Indian Journal of Pathology and Bacteriology, 6, 353. 